\title{
Robust Consumption and Portfolio Choice for Time Varying Investment Opportunities
}

\author{
Hening $\mathrm{Liu}^{\dagger}$ \\ University of Manchester
}

May 20, 2010

\begin{abstract}
This paper examines a continuous-time intertemporal consumption and portfolio choice problem for an investor with recursive preferences. The investor worries about model misspecification and seeks robust decision rules. The expected excess return of a risky asset follows a mean-reverting process. I find that whether the concern about model misspecification decreases the total demand for equities largely depends on risk aversion and the attitude toward intertemporal substitution. When the elasticity of intertemporal substitution is about 1 and risk aversion is moderate, the aversion to model uncertainty increases the proportion of wealth invested in equities. The calibration analysis based on detection-error probabilities shows that the quantitative effect of robustness is almost negligible.
\end{abstract}

JEL: G11; D81; C61

Keywords: robust control, mean-reverting, portfolio choice, recursive preferences

\footnotetext{
$\dagger$ I am grateful to Evan Anderson for constant support and guidance. All errors are my own. Corresponding address: Accounting and Finance Group, Manchester Business School, University of Manchester, Booth Street West, Manchester M15 6PB, UK. E-mail: Hening.Liu@mbs.ac.uk. Telephone: +44(0)16127 54492. Fax: +44(0)16127 54023
} 


\section{Introduction}

Following the seminal work of Merton (1971), a number of papers have examined dynamic portfolio choice when investment opportunities are time varying. To name a few, Kim and Omberg (1996), Campbell and Viceira (1999) and Campbell et al. (2004) analyze intertemporal consumption and portfolio choice when the risk premium of a risky asset follows a mean-reverting process. In those models, the intertemporal hedging demand, which is driven by stochastic variation in investment opportunities, plays an important role in portfolio decisions. The bulk of this literature assumes that investors have complete confidence in the model of asset returns and do not worry about model uncertainty. In recent years, a growing body of literature (e.g., Anderson, Hansen and Sargent, 2000; Hansen and Sargent, 2001 and Maenhout, 2004) begins to analyze implications of model uncertainty for asset pricing and portfolio choice. Model uncertainty refers to the uncertainty about the probability law governing the dynamics of state variables. The well-known Ellsberg paradox suggests that people are averse to model uncertainty. Maenhout (2006) employs the robust control approach of Anderson et al. (2000) to examine intertemporal portfolio choice under model uncertainty for the constant relative risk aversion (CRRA) utility function over terminal wealth. He finds that a concern about model uncertainty lowers the total equity demand in the presence of mean-reverting returns, even when the intertemporal hedging demand is accounted for.

The contribution of this paper is to take into account not only time variation in investment opportunities but also model uncertainty and to analyze intertemporal consumption and portfolio choice for an investor with recursive preferences and an infinite horizon. In particular, as in Campbell et al. (2004), I assume that an investor has Duffie and Epstein's (1992) recursive preferences and the expected returns of a risky asset follows a mean-reverting process. Furthermore, the investor worries that the model describing investment opportunities is subject to model misspecification in the spirit of Anderson et al. (2000) and thus the investor is willing to seek robust consumption and portfolio choice. The model presented here is an extension of the robust portfolio choice model of Maenhout 
(2006) by disentangling the inverse relationship between the coefficient of relative risk aversion and the elasticity of intertemporal substitution (EIS), which is implied by CRRA preferences. Similar to Campbell et al. (2004), I obtain an exact analytical solution when the EIS is equal to 1 and an approximate analytical solution for the EIS different from 1. The key finding of this paper is formalized in terms of an observational equivalence result: the optimal consumption and portfolio decision rules for an uncertainty-averse investor are observationally equivalent to those for an investor who does not worry about model uncertainty but has an enhanced level of effective risk aversion 1 Thus, with the separation between risk aversion and the attitude toward intertemporal substitution, recent empirical asset pricing findings that suggest high estimates of the risk aversion coefficient (relative to the typical empirical estimates from the microeconomic data) can be reconciled with models where there is a preference for robustness $2^{2}$

An important implication of this paper is that a concern about model uncertainty can increase the total equity demand when returns of the risky asset and its expected returns are negatively correlated. This result stands in contrast to the findings of Maenhout (2006). The difference arises because once the separation between risk aversion and the attitude toward intertemporal substitution is achieved and the EIS parameter is controlled for, an increase in effective risk aversion can lead to a significant increase in the intertemporal hedging demand while reduces the mean-variance efficient equity allocation. When the former effect becomes dominant, the desire for robustness increases the total equity demand. Using numerical examples, I show that this occurs for a moderately risk-averse investor when the EIS is about 1. In addition, I use the technique of detection-error probabilities developed by Anderson et al. (2000) and the methodology for calculating detection-error probabilities by Maenhout (2006) to empirically assess the quantitative effect of model uncertainty for the sample data of Campbell et al. (2004). I show that the strength of the quantitative effect of model uncertainty reaches an almost negligible level for various empirically plausible values of the preference parameters.

1 Maenhout (2004) shows the observational equivalence for recursive preferences in an i.i.d. case. Maenhout (2006) shows the observational equivalence for CRRA utility in the case of mean reversion.

2 See, for example, Bansal and Yaron (2004) and Vissing-Jørgensen and Attanasio (2003). 
This result echoes the finding of Maenhout (2006) that given the same data sample, the shrunk scope of robustness in the presence of mean reversion is largely caused by the effect of hedging demand, which confronts a robust investor an easier model selection problem than in the i.i.d. case. Here, because the horizon is assumed to be infinite, the importance of hedging demand in portfolio decisions is magnified. As a consequence, the scope of robustness becomes even smaller, as compared to Maenhout (2006).

The rest of the paper is organized as follows. Section 2 presents the dynamic optimization problem for a robust investor with recursive preferences. Section 3 gives an exact analytical solution and an approximate analytical solution when expected returns follow a mean-reverting process. Section 4 describes the calculation of detection-error probabilities based on the Radon-Nikodym derivatives. Section 5 explores the quantitative effect of robustness by calibrating the model to the market data. Section 6 concludes. The Appendix contains the solutions to the intertemporal consumption and portfolio choice and the proof of the main proposition of the paper.

\section{The Investor's Robust Optimization Problem}

I assume that there are two assets available for investment, a riskless short-term bond paying instantaneous return $r$ and a risky asset with the following price dynamics:

$$
d S_{t}=S_{t} \mu_{t} d t+S_{t} \sigma d B_{t}
$$

The instantaneous expected return $\mu_{t}$ is assumed to follow a mean-reverting process:

$$
d \mu_{t}=\kappa\left(\bar{\mu}-\mu_{t}\right) d t+\sigma_{\mu} \rho d B_{t}+\sigma_{\mu} \sqrt{1-\rho^{2}} d \hat{B}_{t}
$$

where $\kappa, \bar{\mu}$ and $\sigma_{\mu}$ are all positive constants, $\rho$ is the correlation coefficient $\operatorname{corr}(d S, d \mu)$ and $\hat{B}_{t}$ and $B_{t}$ are independent Brownian motions. 
The investor's wealth dynamics can be described by the following stochastic differential equation $(\mathrm{SDE}):$

$$
d W_{t}=\left[W_{t}\left(r+\alpha_{t}\left(\mu_{t}-r\right)\right)-C_{t}\right] d t+W_{t} \alpha_{t} \sigma_{S} d B_{t}
$$

where $\alpha_{t}$ is the proportion of wealth $W_{t}$ invested in the risky asset and $C_{t}$ is consumption at time $t$.

I assume that the investor has recursive preferences over consumption and the preferences take Duffie and Epstein's (1992) continuous-time parameterization. In the absence of a preference for robustness, the parameterization has the following form

$$
V_{t}=\int_{t}^{\infty} f\left(C_{s}, V_{s}\right) d s
$$

where $f(C, V)$ is a normalized aggregator of current consumption and continuation utility that is given by

$$
f(C, V)=\frac{\beta}{1-\frac{1}{\psi}}(1-\gamma) V\left[\left(\frac{C}{((1-\gamma) V)^{\frac{1}{1-\gamma}}}\right)^{\left(1-\frac{1}{\psi}\right)}-1\right]
$$

Here, $\beta$ is the rate of time preference, $\gamma$ is the coefficient of relative risk aversion and $\psi$ is the elasticity of intertemporal substitution (EIS). With the restriction $\psi=1 / \gamma$, the normalized aggregator (4) reduces to the standard additive power utility function, for which log utility obtains for $\gamma=\psi=1$. When $\psi=1$, the aggregator $f(C, V)$ takes the form

$$
f(C, V)=\beta(1-\gamma) V\left[\log (C)-\frac{1}{1-\gamma} \log ((1-\gamma) V)\right]
$$

In the absence of model uncertainty, the investor's problem is

$$
\max _{\left\{C_{t}, \alpha_{t}\right\}} E_{0}\left[\int_{0}^{\infty} f\left(C_{s}, V_{s}\right) d s\right]
$$

subject to (2) and (3). This optimization problem has been analyzed by Campbell et al. (2004). The 
Hamilton-Jacobi-Bellman (HJB) equation for this problem is

$$
0=\sup _{\alpha_{t}, C_{t}}\left\{f\left(C_{t}, V_{t}\right)+\mathcal{D}^{(C, \alpha)} V\right\}
$$

where $\mathcal{D}^{(C, \alpha)} V$ is the infinitesimal generator applied to the value function $V$ and is defined by

$$
\begin{aligned}
\mathcal{D}^{(C, \alpha)} V= & V_{W}\left[W_{t}\left(r+\alpha_{t}\left(\mu_{t}-r\right)\right)-C_{t}\right]+V_{\mu} \kappa\left(\bar{\mu}-\mu_{t}\right) \\
& +\frac{1}{2} V_{W W}\left(\alpha_{t} \sigma_{S} W_{t}\right)^{2}+V_{W \mu} W_{t} \alpha_{t} \rho \sigma_{S} \sigma_{\mu}+\frac{1}{2} V_{\mu \mu} \sigma_{\mu}^{2} .
\end{aligned}
$$

Intuitively, $\mathcal{D}^{(C, \alpha)} V$ is a measure of the instantaneous expected continuation value in the Bellman equation. As shown below, the adjustment to this term reflects the concern about model uncertainty. A robust investor deems the state dynamics (2) and (3) as only an approximation and possibly misspecified. The investor partly accepts the usefulness of this reference model but still doubts its validity. He therefore wants to consider a family of alternative models that are close to and hard to distinguish from the reference model.

Denote the state vector $Y_{t} \equiv\left(W_{t}, \mu_{t}\right)^{\top}$. The reference model can then be written as

$$
d Y_{t}=\Theta\left(Y_{t}\right) d t+\Lambda\left(Y_{t}\right) d \mathbf{B}_{t}
$$

where $\Theta$ is the drift vector, $\Lambda$ is the volatility matrix of the state vector $Y_{t}, \Sigma=\Lambda \Lambda^{\top}$ is the covariance matrix and $\mathbf{B}$ is the Brownian vector $\mathbf{B}_{t} \equiv\left(B_{t}, \hat{B}_{t}\right)^{\top}$. The vector $\Theta$ and the matrix $\Lambda$ have the appropriate functional forms inherited from (2) and (3). The distorted law of motion of the state vector implied by an alternative model is

$$
d Y_{t}=\Theta\left(Y_{t}\right) d t+\Lambda\left(Y_{t}\right)\left[\Lambda\left(Y_{t}\right)^{\top} u\left(Y_{t}\right) d t+d \mathbf{B}_{t}\right]
$$

where $u\left(Y_{t}\right)$ is an endogenous drift adjustment vector to be determined from solving a robust opti- 
mization problem presented below. Thus, model uncertainty indeed concerns the uncertainty about the drift functions of the state variables. Suppose $\mathbb{P}$ is the subjective probability measure under the reference model and $\mathbb{Q}$ under the alternative model. It follows from Girsanov's theorem that the Radon-Nikodym derivative is

$$
\left(\frac{d \mathbb{Q}}{d \mathbb{P}}\right)_{t}=\Xi_{1, t}
$$

where

$$
\frac{d \Xi_{1, t}}{\Xi_{1, t}}=\Lambda\left(Y_{t}\right)^{\top} u\left(Y_{t}\right) d \mathbf{B}_{t}, \quad \Xi_{1,0}=1
$$

The interpretation is that the investor endogenously chooses an alternative belief about the dynamics of the state variables and accordingly the optimal consumption and portfolio policies.

The investor worries that an alternative model has an adverse effect on the expected continuation payoff and wants to consider this worst-case alternative model in making decisions. The investor is willing to consider alternative models that are close to the reference model. In the meantime, a penalty term should be incurred to penalize the distance of an alternative model to the reference model. Within the framework of Anderson et al. (2000) and Hansen et al. (2006), a robust investor solves the following optimization problem ${ }^{3}$

$$
\max _{\left\{C_{t}, \alpha_{t}\right\}} E_{0}\left[\int_{0}^{\infty}\left[f\left(C_{s}, V_{s}\right)+e^{-\beta s} \frac{1}{2 \eta} u^{\top} u\right] d s\right]
$$

where the second term is discounted relative entropy that quantifies the penalty term. The HJB equation is

$$
0=\sup _{\alpha_{t}, C_{t}} \inf _{u}\left\{f\left(C_{t}, V_{t}\right)+\mathcal{D}^{(C, \alpha)} V+u^{\top} \Sigma \partial V+\frac{1}{2 \eta} u^{\top} \Sigma u\right\}
$$

The third term in (8) reflects the adjustment to the expected continuation value when the state dynamics are governed by the alternative model (5). This term also captures the adverse effect of

3 This is the multiplier formulation of the robust control framework. With the restriction $\psi=1 / \gamma$, this formulation belongs to the time-separable case of Anderson et al. (2000) and Hansen et al. (2006). 
model misspecification on the continuation value. As for the relative entropy term, alternative models that are close to the reference model have low entropy and low log-likelihood ratios. These models are therefore statistically difficult to distinguish from the reference model.

The preference parameter $\eta \geq 0$ measures the strength of preference for robustness or the degree of confidence in the reference model. When $\eta=0$, the investor has complete confidence in the reference model. This case is identical to that considered by Campbell et al. (2004).

\section{The Robust Intertemporal Consumption and Portfolio Decisions}

I impose the set of admissible portfolio strategies with well-defined utility, namely, set $\mathcal{A}$ given by

$$
\mathcal{A}=\left\{\alpha: W_{t} \geq 0 \text { and } \mathbb{E}^{\mathbb{Q}}\left[f\left(C_{t}, V_{t}\right)<\infty\right] \text { for all } t \geq 0,\right\}
$$

The first order conditions (FOCs) with respect to $u$ are

$$
u^{*}=-\eta \partial V
$$

The endogenous drift distortion depends on the state variables through the partial derivatives of the value function in an implicit way. Unless a complete solution to the problem is obtained, the dependence cannot be explicitly characterized.

Substituting the FOCs back into the HJB equation gives

$$
0=\sup _{\alpha_{t}, C_{t}}\left\{f\left(C_{t}, V_{t}\right)+\mathcal{D}^{(C, \alpha)} V-\frac{\eta}{2}\left[\left(W_{t} \alpha_{t} \sigma_{S}\right)^{2} V_{W}^{2}+2 W_{t} \alpha_{t} \rho \sigma_{S} \sigma_{\mu} V_{W} V_{\mu}+\sigma_{\mu}^{2} V_{\mu}^{2}\right]\right\}
$$

As noted by Anderson et al. (2000) and Maenhout (2006), robustness leads to an adjustment term in the HJB equation that reflects a concern about the quadratic variation in the partial derivatives of the value function weighted by the preference parameter $\eta$. 
The first order conditions for consumption are

$$
\begin{array}{rlrl}
C_{t} & =V_{W}^{-\psi}[(1-\gamma) V]^{\frac{1-\gamma \psi}{1-\gamma}} \beta^{\psi} & \text { when } \psi \neq 1 \\
C_{t}=\frac{V}{V_{W}}(1-\gamma) \beta & \text { when } \psi=1
\end{array}
$$

The first order condition for portfolio choice is

$$
\alpha_{t}=\frac{V_{W}\left(\mu_{t}-r\right)}{\left(-V_{W W} W_{t}+\eta V_{W}^{2} W_{t}\right) \sigma_{S}^{2}}+\frac{V_{W \mu} \rho \sigma_{\mu}}{\left(-V_{W W} W_{t}+\eta V_{W}^{2} W_{t}\right) \sigma_{S}}+\frac{-\eta \rho \sigma_{\mu} V_{W} V_{\mu}}{\left(-V_{W W} W_{t}+\eta V_{W}^{2} W_{t}\right) \sigma_{S}}
$$

To explicitly solve the model, I follow Maenhout $(2004,2006)$ and impose the homothetic robustness specification. In particular, I assume that the preference parameter $\eta$ is state-dependent and scaled by the value function 4 .

$$
\eta\left(W_{t}, \mu_{t}\right)=\frac{\phi}{(1-\gamma) V\left(W_{t}, \mu_{t}\right)}>0
$$

where the parameter $\phi$ can be interpreted as the preference for robustness or uncertainty aversion. The HJB equation then becomes

$$
\begin{aligned}
0= & \sup _{\alpha_{t}, C_{t}}\left\{f\left(C_{t}, V_{t}\right)+V_{W}\left[W_{t}\left(r+\alpha_{t}\left(\mu_{t}-r\right)\right)-C_{t}\right]+V_{\mu} \kappa\left(\bar{\mu}-\mu_{t}\right)\right. \\
& +\frac{1}{2} V_{W W}\left(\alpha_{t} \sigma_{S} W_{t}\right)^{2}+V_{W \mu} W_{t} \alpha_{t} \rho \sigma_{S} \sigma_{\mu}+\frac{1}{2} V_{\mu \mu} \sigma_{\mu}^{2} \\
& \left.-\left(\frac{\phi}{2(1-\gamma)} \frac{\alpha_{t}^{2} \sigma_{S}^{2} W_{t}^{2} V_{W}^{2}}{V}+\frac{\phi}{1-\gamma} \frac{W_{t} \alpha_{t} \rho \sigma_{S} \sigma_{\mu} V_{W} V_{\mu}}{V}+\frac{\phi}{2(1-\gamma)} \frac{\sigma_{\mu}^{2} V_{\mu}^{2}}{V}\right)\right\} .
\end{aligned}
$$

For different values of the EIS parameter $(\psi=1$ or $\psi \neq 1)$, the HJB equation results in different partial differential equations because different normalized aggregators are used. I show these equations, respectively, for each of the two cases in Appendix. When $\psi=1$, the HJB equation above admits an

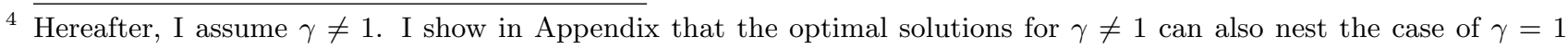
except that the value functions take different forms. 
exact analytical solution of the form

$$
V\left(W_{t}, \mu_{t}\right)=\left(I\left(\mu_{t}\right)\right)^{1-\gamma} \frac{W_{t}^{1-\gamma}}{1-\gamma}
$$

with

$$
I\left(\mu_{t}\right)=\exp \left\{D+E \mu_{t}+\frac{G}{2} \mu_{t}^{2}\right\}
$$

where $D, E$ and $G$ satisfy a system of recursive equations given in Appendix and are functions of the primitive parameters of the model describing investment opportunities and preferences. The optimal consumption and portfolio choice are given by

$$
\begin{gathered}
C_{t}=\beta W_{t} \\
\alpha_{t}=\frac{\mu_{t}-r}{(\gamma+\phi) \sigma_{S}^{2}}+\frac{1-\gamma-\phi}{\gamma+\phi} \frac{\rho \sigma_{\mu}}{\sigma_{S}}\left(E+G \mu_{t}\right) .
\end{gathered}
$$

In the more general case of $\psi \neq 1$, there is no exact analytical solution to (8). But I can use the log-linear approximation method described in Campbell et al. (2004) to solve for an approximate analytical solution 5 When $\psi \neq 1$ and $\gamma \neq 1$, the solution to the value function is given by

$$
V\left(W_{t}, \mu_{t}\right)=\left(K\left(\mu_{t}\right)\right)^{-\frac{1-\gamma}{1-\psi}} \frac{W_{t}^{1-\gamma}}{1-\gamma}
$$

where $K\left(\mu_{t}\right)$ satisfies a nonlinear ordinary differential equation given in Appendix. Applying loglinear approximation, the approximate analytical solution to $K\left(\mu_{t}\right)$ is given by

$$
K\left(\mu_{t}\right)=\exp \left\{\hat{D}+\hat{E} \mu_{t}+\frac{\hat{G}}{2} \mu_{t}^{2}\right\}
$$

5 I obtain the approximate analytical solution using a numerical recursive procedure suggested in Campbell and Viceira (1999). Appendix describes the details of the procedure. The procedure converges extremely rapidly for the parameter values used in the calibration analysis of this paper. See Campbell and Viceira (1999) for a related discussion on the convergence properties. 
where $\hat{D}, \hat{E}$ and $\hat{G}$ satisfy a system of recursive equations shown in Appendix and are functions of the primitive parameters of the model describing investment opportunities and preferences. The optimal portfolio choice is given by

$$
\alpha_{t}=\frac{\mu_{t}-r}{(\gamma+\phi) \sigma_{S}^{2}}-\frac{1}{1-\psi} \frac{1-\gamma-\phi}{\gamma+\phi} \frac{\rho \sigma_{\mu}}{\sigma_{S}}\left(\hat{E}+\hat{G} \mu_{t}\right)
$$

The following proposition establishes the link of the solutions here to those of Campbell et al. (2004) where there is no model uncertainty.

Proposition 1 Suppose investment opportunities are described by (1)-(2), an uncertainty-averse investor with a homothetic preference for robustness $\eta(W, \mu)=\frac{\phi}{(1-\gamma) V(W, \mu)}$ and recursive preferences is observationally equivalent to an investor with recursive preferences and effective risk aversion $\gamma+\phi$

Proof. See Appendix.

The above proposition extends the result of Maenhout (2006) to the case of recursive preferences. The observational equivalence holds not only for the optimal portfolio choice but also for the optimal consumption-to-wealth ratio when the separation between risk aversion and intertemporal substitution is allowed for. In the optimal portfolio rules (16) and (18), model uncertainty affects both myopic demand and the intertemporal hedging demand ${ }^{6}$ Under recursive preferences, Maenhout (2004) derives the first term in (16) (or 18) ) as the optimal portfolio rule for constant investment opportunities. Thus, intertemporal substitution does not affect robust portfolio decisions. Furthermore, this implies that a high level of uncertainty aversion always leads to a low equity demand for risk-averse investors. I show below that this is not true for time-varying investment opportunities. Maenhout (2006) assumes a mean-reverting risk premium and finds that having $\phi>0$ lowers the optimal equity demand for a CRRA utility investor. Recursive preferences nest CRRA utility as

6 It is difficult to separate from the total hedging demand a hedging component in analytical form that is solely attributed to model uncertainty because the uncertainty aversion parameter $\phi$ also appears in the recursive equations for $D(\hat{D})$, $E(\hat{E})$ and $G(\hat{G})$. 
a special case and distinguish intertemporal substitution from risk aversion. This distinction can generate important implications concerning the effect of robustness on portfolio choice. In Section 5, I show these results using numerical examples.

\section{Detection-Error Probabilities}

Our task is to calibrate the price dynamics (1)-(2) to the market data and use the optimal portfolio rules derived in the previous section to find the quantitative effect of model uncertainty. I employ the statistical model-detection tool in the form of detection-error probabilities suggested by Anderson et al. (2000) to calibrate the uncertainty aversion parameter. According to Anderson et al. (2000), a reasonable value for uncertainty aversion $\phi$ should render the corresponding worst-case alternative model sufficiently close to the reference model based on a time series sample with finite length. A high detection-error probability indicates a high likelihood that a robust investor will make a mistake when choosing between the worst-case alternative model and the reference model. The parameter values of $\phi$ should be chosen in such a way that the detection-error probability is high enough for the investor to face a difficult model selection problem.

I employ the methodology of Maenhout (2006) to calculate detection-error probabilities in the presence of mean reversion 4 The solutions to the intertemporal consumption and portfolio problem

7 Maenhout (2006) develops a methodology for calculating detection-error probabilities based on Fourier inversion of the conditional characteristic functions of the Radon-Nikodym derivatives between the worst-case model and the reference model. He shows that the conditional characteristic functions can be characterized by systems of ordinary differential equations (ODEs) with time-varying and complex coefficients. Here, the horizon is infinite and the optimal decision rules are stationary. This simplification allows me to derive exact analytical solutions to the conditional characteristic functions of the Radon-Nikodym derivatives. For ease of notation, the technical details are not included but are available upon request from the author. 
(8) imply that the worst-case alternative model (model $\mathbb{Q}$ hereafter) has the following form:

$$
\begin{aligned}
d S_{t}= & {\left[\mu_{t}-\frac{\phi}{\gamma+\phi}\left[\left(\mu_{t}-r\right)+\left(\mathcal{E}_{j}+\mathcal{G}_{j} \mu_{t}\right) \rho \sigma_{\mu} \sigma_{S}\right]\right] S_{t} d t+\sigma_{S} S_{t} d B_{t} } \\
d \mu_{t}= & {\left[\kappa\left(\bar{\mu}-\mu_{t}\right)-\frac{\phi}{\gamma+\phi} \frac{\mu_{t}-r}{\sigma_{S}} \rho \sigma_{\mu}-\phi\left(1+\frac{1-\gamma-\phi}{\gamma+\phi} \rho^{2}\right)\left(\mathcal{E}_{j}+\mathcal{G}_{j} \mu_{t}\right) \sigma_{\mu}^{2}\right] } \\
& +\sigma_{\mu} \rho d B_{t}+\sigma_{\mu} \sqrt{1-\rho^{2}} d \hat{B}_{t} \\
j= & 0,1
\end{aligned}
$$

where $\mathcal{E}_{0}=E, \mathcal{G}_{0}=G$ corresponds to the case $\psi=1$, and $\mathcal{E}_{1}=-\frac{\hat{E}}{1-\psi}, \mathcal{G}_{1}=-\frac{\hat{G}}{1-\psi}$ corresponds to the case $\psi \neq 1$. Not surprisingly, the implied drift distortions rely on the state variables not only explicitly but also implicitly through the intertemporal portfolio choice rule. Under recursive preferences, the risk aversion parameter $\gamma$ and the EIS parameter $\psi$ are both free parameters affecting the implied drift distortions. Similar to Maenhout (2006), the drift distortions include a myopic distortion and an intertemporal hedging distortion, captured respectively by the first term and the second term of the drift distortions in $(19)$ and $(20)$.

The column vector $g$, defined as $g \equiv \Lambda^{\top} u$ is given by

$$
g\left(\mu_{t}\right)=-\phi\left[\begin{array}{c}
\frac{1}{\gamma+\phi}\left(\frac{\mu_{t}-r}{\sigma_{S}}+\left(\mathcal{E}_{j}+\mathcal{G}_{j} \mu_{t}\right) \rho \sigma_{\mu}\right) \\
\sigma_{\mu} \sqrt{1-\rho^{2}}\left(\mathcal{E}_{j}+\mathcal{G}_{j} \mu_{t}\right)
\end{array}\right]
$$

with $j=0,1$. It follows from (6) and (7) that the $\log$ of the Radon-Nikodym derivative of the worst-case probability measure $\mathbb{Q}$ with respect to the reference measure $\mathbb{P}$ is

$$
\zeta_{1, t} \equiv \log \Xi_{1, t}=-\int_{0}^{t} g\left(\mu_{s}\right)^{\top} d \mathbf{B}_{s}-\frac{1}{2} \int_{0}^{t} g\left(\mu_{s}\right)^{\top} g\left(\mu_{s}\right) d s
$$

The log-likelihood ratio therefore follows a stochastic process with time-varying drift and volatility that are functions of the preference parameters and the parameters describing investment opportu- 
nities. Similarly, the log of the Radon-Nikodym derivative $\Xi_{2, t} \equiv \frac{d \mathbb{P}}{d \mathbb{Q}}$ of the probability measure of model $\mathbb{P}$ with respect to that of model $\mathbb{Q}$ is

$$
\zeta_{2, t} \equiv \log \Xi_{2, t}=\int_{0}^{t} g\left(\mu_{s}\right)^{\top} d \mathbf{B}_{s}+\frac{1}{2} \int_{0}^{t} g\left(\mu_{s}\right)^{\top} g\left(\mu_{s}\right) d s
$$

Suppose model $\mathbb{P}$ is true, the investor will mistakenly reject it in favor of model $\mathbb{Q}$ based on a finite sample with length $N$ when $\zeta_{1, N}>0$. Conversely, if model Q is correct, it will be rejected erroneously when $\zeta_{2, N}>0$. Let $\left\{\mathcal{F}_{t}: t \geq 0\right\}$ be the filtration with respect to which expectations and probabilities are conditioned. The detection-error probability $\varepsilon_{N}(\phi)$, which is the time- 0 conditional probability of choosing a wrong model based on a finite sample of length $N$, is defined as (see Maenhout 2004, 2006)

$$
\varepsilon_{N}(\phi) \equiv \frac{1}{2} \operatorname{Pr}\left(\zeta_{1, N}>0 \mid \mathbb{P}, \mathcal{F}_{0}\right)+\frac{1}{2} \operatorname{Pr}\left(\zeta_{2, N}>0 \mid \mathbb{Q}, \mathcal{F}_{0}\right)
$$

This definition highlights the dependence of the detection-error probability on the uncertainty aversion parameter $\phi$. As $\phi$ increases, the investor desires more robustness. In this case, the worst-case alternative model looks more differently from the reference model and the model selection problem becomes easier for the investor, resulting in a lower detection-error probability.

\section{Calibration and Results}

Parameter estimates for calibration are drawn from Campbell et al. (2004) (the quarterly data from 1947 to 1995) and displayed in Table 1. Given the observational equivalence result, the effect of an increase in effective risk aversion can be reinterpreted as the effect of a preference for robustness. Figure 1 summarizes the effects of model uncertainty on the mean optimal equity allocation and the mean hedging equity allocation for different values of the EIS parameter. In particular, I consider a moderate level of risk aversion $\gamma=1.5$. In contrast to the previous finding of Maenhout (2006) that model uncertainty always reduces the total equity demand when $\gamma$ is greater than 1 , I show 
that the total equity demand can increase in response to the desire for robustness. This occurs when the EIS parameter is about $1(\psi=1 / 0.75$ and 1 in Figure 1$)$. The notable difference from the result of Maenhout (2006) stems from recursive preferences that allow for the separation between risk aversion and intertemporal substitution. To reconcile the seeming contradiction, I argue that Maenhout's results are partly driven by the effect of intertemporal substitution, not entirely by the effect of robustness. On the one hand, concerns for robustness decreases the myopic equity demand. On the other hand, given negative correlation between stock returns and innovations in expected future returns, equities can be used as a tool of hedging against the variation in expected returns. An investor desiring robustness will optimally increase the equity demand due to hedging concerns since robustness imputes a strong preference for smoothing utility across states. When this effect is strong enough, it will counteract the effect of model uncertainty on myopic demand and result in a higher total equity demand. More important, the impact of model uncertainty on hedging demand does depend on the willingness to substitute intertemporally. As shown in Figure 1 (right panel), model uncertainty has a mitigated effect on hedging demand for low values of the EIS parameter (e.g., $\psi=1 / 1.5$ and 1/20) $!^{8}$ The CRRA utility function implies that the EIS parameter and the coefficient of relative risk aversion are inversely related $(\psi=1 / \gamma)$. A high risk aversion implies a low willingness to substitute intertemporally, in which case the effect of model uncertainty on hedging demand may be dominated by that on myopic demand. As a result, the net effect is to reduce the optimal equity demand, as observed by Maenhout (2006). This corresponds to the case $(\gamma=1.5, \psi=1 / 1.5)$ in Figure 1. By disentangling risk aversion and intertemporal substitution, the effect of intertemporal substitution is controlled for and thus the effect of model uncertainty obtains in a clean manner.

\section{[Insert Table 1 here]}

\section{[Insert Figure 1 here]}

8 An investor who is unwilling to substitute intertemporally wants to maintain a stable consumption growth rate and only has modest motive to hedge against the variation in expected future returns. See Campbell and Viceira (1999) for related results and discussions. 
Finally, I investigate the extent of the impact of robustness based on detection-error probabilities. Anderson et al. (2000) advocate a threshold of $10 \%$ for the detection-error probability $\varepsilon_{N}(\phi)$. A value of $\varepsilon_{N}(\phi)$ lower than the threshold is of little practical interest because the decision-maker can hardly make a mistake in model selection.

Table 2 reports $\varepsilon_{N}(\phi)$ for $\gamma=\{0.75,1,1.5,2,4,6\}$ and $\psi=\left\{\frac{1}{0.75}, 1, \frac{1}{1.5}, \frac{1}{2}, \frac{1}{4}, \frac{1}{6}\right\}$ for the sample of Campbell et al. (2004). The values of the uncertainty aversion parameter $\phi$ are chosen such that $\varepsilon_{N}(\phi)$ stays beyond the threshold in most cases. A notable result is that there is little room for model uncertainty to exist. To obtain meaningful detection-error probabilities, the postulated uncertainty aversion parameter is of a lower order of magnitude relative to the risk aversion parameter $\gamma$. For example, when $\gamma=4$ and $\psi=1 / 0.75$, the concern about model uncertainty can at best support a value of $\phi$ that is about $\gamma / 15$, which implies an effective risk aversion of $\gamma \times 1.067$. These findings strengthen the result of Maenhout (2006) that when returns display mean reversion and investment opportunities are fully observable, model detection becomes very easy and the scope of model uncertainty shrinks sharply relative to the i.i.d. case. Here, the scope of model uncertainty becomes even smaller than in Maenhout (2006) because the investor has an infinite horizon while Maenhout (2006) assumes finite horizons $9^{9}$ When the risk premium is mean-reverting, investors with an infinite horizon substantially increase hedging demand and the amount of wealth invested in the risky asset 10 According to Maenhout (2006), the robust portfolio choice problem can be reinterpreted as a two-player game in which a fictitious agent chooses the worst-case model and the distortion between the worst-case model and the reference model is an increasing function of the portfolio rule chosen by a robust investor 11 When the horizon is infinite, the magnified hedging demand translates into a model distortion that is large in magnitude and therefore more easily detectable. Thus, the scope of model uncertainty

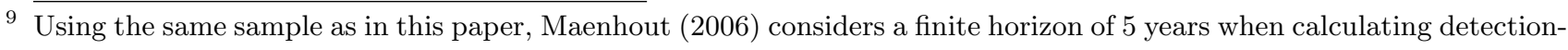
error probabilities.

${ }^{10}$ Several studies, to name a few, Kim and Omberg (1996) and Wachter (2002), find that the optimal equity allocation is monotonically increasing in the investment horizon.

${ }^{11}$ Maenhout (2006) argues that the continuous observability of the state variable is one of the major reasons for easy model detection in non-i.i.d. cases with a fully observable investment opportunity set. The continuous observability is manifested through the hedging component and the myopic component in drift distortions that are nonlinear functions of the state variable. 
shrinks considerably for various values of the preference parameters $\gamma$ and $\psi$ as compared to the finite horizon case of Maenhout (2006). In addition, Table 2 also shows that the detection-error probability also depends on the EIS parameter. In particular, a high $\psi$ is associated with low values of $\varepsilon_{N}(\phi)$. As noted by Campbell and Viceira (1999), investors who are more willing to substitute intertemprally invest more in equities due to hedging motives, all else being equal. This leads to easy model detection and small scope of model uncertainty when the EIS parameter is high.

\section{[Insert Table 2 here]}

\section{Conclusion}

I have examined a continuous-time intertemporal consumption and portfolio choice problem in which investment opportunities are time-varying and the roles of risk aversion, uncertainty aversion and intertemporal substitution are separated among one another. The investor worries that his model of asset returns may be misspecified and wants robust consumption and portfolio decision rules that take into account model uncertainty. I find that uncertainty aversion can increase the total equity demand for moderately risk-averse investor when the elasticity of intertemporal substitution is about 1. Using the technique of detection-error probabilities, I calibrate the quantitative effect of model uncertainty and find that the scope of model uncertainty is negligible under mean-reverting and fully observable investment opportunities.

\section{Appendix}

The Solution for $\psi=1$. Substitution of (14) into the first order conditions for consumption and portfolio choice (11) and (12) leads to the optimal consumption policy $C^{*}=\beta W$ and the optimal portfolio rule

$$
\alpha_{t}=\frac{\mu_{t}-r}{(\gamma+\phi) \sigma_{S}^{2}}+\frac{1-\gamma-\phi}{\gamma+\phi} \frac{\rho \sigma_{\mu}}{\sigma_{S}}\left(E+G \mu_{t}\right)
$$


Substituting (14) and (15) into the HJB equation (9), I obtain the following equation after simplification:

$$
\begin{aligned}
0= & \beta \log \beta-\beta\left(D+E \mu_{t}+\frac{G}{2} \mu_{t}^{2}\right)-\beta+r+\left(\mu_{t}-r\right) \alpha_{t}+\kappa\left(\bar{\mu}-\mu_{t}\right)\left(E+G \mu_{t}\right)+\frac{\sigma_{\mu}^{2}}{2} G \\
& +\frac{\sigma_{\mu}^{2}}{2}[1-(\gamma+\phi)]\left(E+G \mu_{t}\right)^{2}-\frac{1}{2}(\gamma+\phi) \sigma_{S}^{2} \alpha_{t}^{2}+[1-(\gamma+\phi)] \rho \sigma_{S} \sigma_{\mu} \alpha_{t}\left(E+G \mu_{t}\right) .
\end{aligned}
$$

Collecting terms in $\mu_{t}^{2}, \mu_{t}$ and constant terms, I can obtain $D, E$ and $G$ as the solution to the following system of recursive equations:

$$
\begin{aligned}
0= & \frac{1}{2(\gamma+\phi) \sigma_{S}^{2}}+\left(-\frac{\beta}{2}-\kappa+\frac{1-(\gamma+\phi)}{\gamma+\phi} \frac{\rho \sigma_{\mu}}{\sigma_{S}}\right) G \\
& +\frac{\sigma_{\mu}^{2}}{2}\left\{[1-(\gamma+\phi)]+\frac{(1-(\gamma+\phi))^{2}}{\gamma+\phi} \rho^{2}\right\} G^{2} \\
0= & -\frac{r}{(\gamma+\phi) \sigma_{S}^{2}}+\left(\kappa \bar{\mu}-\frac{1-(\gamma+\phi)}{\gamma+\phi} \frac{\rho \sigma_{\mu}}{\sigma_{S}}\right) G \\
& +\left\{-\kappa-\beta+\frac{1-(\gamma+\phi)}{\gamma+\phi} \frac{\rho \sigma_{\mu}}{\sigma_{S}}+\sigma_{\mu}^{2}\left[1-(\gamma+\phi)+\frac{(1-(\gamma+\phi))^{2}}{\gamma+\phi} \rho^{2}\right] G\right\} E \\
0= & \beta(\log \beta-1)+r+\frac{r^{2}}{2(\gamma+\phi) \sigma_{S}^{2}}-\beta D+\frac{\sigma_{\mu}^{2}}{2} G+\left(\kappa \bar{\mu}-\frac{1-(\gamma+\phi)}{\gamma+\phi} \frac{\rho \sigma_{\mu}}{\sigma_{S}}\right) E \\
& +\frac{\sigma_{\mu}^{2}}{2}\left(1-(\gamma+\phi)+\frac{(1-(\gamma+\phi))^{2}}{\gamma+\phi} \rho^{2}\right) E^{2} .
\end{aligned}
$$

Equation 22 is a quadratic equation which has two roots. The solution to $G$ is the root associated with the positive discriminant of the equation. This root also ensures that $G=0$ when $\gamma+\phi=1$. The solutions to $D$ and $E$ follow immediately from Equations 23) and 24.

Next, I show that the solution to the optimal portfolio rule also nests the case of logarithmic utility $(\psi=\gamma=1)$. When $\gamma \rightarrow 1$, the preference parameter $\eta$ assumed in 13 converges to $\phi$ based on the solution to the value function when $\gamma \neq 1$ :

$$
\eta_{\log }=\lim _{\gamma \rightarrow 1} \frac{\phi}{W^{1-\gamma} \exp \left[(1-\gamma)\left(D+E \mu_{t}+\frac{G}{2} \mu_{t}^{2}\right)\right]}=\phi
$$


Then it is straightforward to show that the HJB equation (9) can be solved by $V\left(W_{t}, \mu_{t}\right)=\log W+$ $D+E \mu_{t}+\frac{G}{2} \mu_{t}^{2}$, where $D, E$ and $G$ solve the system of recursive equations 222-24 with $\gamma=1$. The optimal portfolio rule is then given by

$$
\alpha_{t}=\frac{\mu_{t}-r}{(1+\phi) \sigma_{S}^{2}}-\frac{\phi}{1+\phi} \frac{\rho \sigma_{\mu}}{\sigma_{S}}\left(E+G \mu_{t}\right)
$$

The Solution for $\psi \neq 1$. Substituting the value function (17) into the HJB equation (9), I obtain, after some simplification, the following ordinary differential equation

$$
\begin{aligned}
0= & -\beta^{\psi} K^{-1}+\beta \psi+r(1-\psi)+\frac{1-\psi}{2(\gamma+\phi)}\left(\frac{\mu_{t}-r}{\sigma_{S}}\right)^{2}-\frac{\sigma_{\mu}^{2}}{2} \frac{K_{\mu \mu}}{K} \\
& +\frac{\sigma_{\mu}^{2}}{2}\left[1+\frac{1-\gamma-\phi}{1-\psi}\left(1+\frac{1-\gamma-\phi}{\gamma+\phi} \rho^{2}\right)\right]\left(\frac{K_{\mu}}{K}\right)^{2} \\
& -\left[\kappa\left(\bar{\mu}-\mu_{t}\right)+\frac{1-\gamma-\phi}{\gamma+\phi} \rho \sigma_{\mu}\left(\frac{\mu_{t}-r}{\sigma_{S}}\right)\right] \frac{K_{\mu}}{K} .
\end{aligned}
$$

This equation does not admit an exact analytical solution because the appearance of the term $\beta^{\psi} K^{-1}$. To this end, I use the log-linear approximation method described in Campbell et al. (2004) to find an approximate analytical solution. Note that the term $\beta^{\psi} K^{-1}$ is exactly the consumption-wealth ratio, which can be approximated around the unconditional mean of the log consumption-wealth ratio as:

$$
\beta^{\psi} K\left(\mu_{t}\right)^{-1} \approx k_{0}+k_{1}\left(\psi \log \beta-k_{t}\right)
$$

with $k_{1}=\exp \left\{\mathbb{E}\left[c_{t}-w_{t}\right]\right\}$ and $k_{0}=k_{1}\left(1-\log k_{1}\right)$ where $c_{t}=\log C_{t}, w_{t}=\log W_{t}, k_{t}=\log K\left(\mu_{t}\right)$. Substituting the approximation (26) for the first term of (25) gives another ODE which admits an exact analytical solution. I conjecture $K\left(\mu_{t}\right)$ is of the exponential-quadratic form

$$
K\left(\mu_{t}\right)=\exp \left\{\hat{D}+\hat{E} \mu_{t}+\frac{\hat{G}}{2} \mu_{t}^{2}\right\} .
$$


Substitution of (27) into ODE (25) with the log-linear approximation (26) transform the Bellman equation into the following equation

$$
\begin{aligned}
0= & -k_{0}-k_{1}\left\{\psi \log \beta-\left(\hat{D}+\hat{E}+\frac{\hat{G}}{2} \mu_{t}^{2}\right)\right\}+\beta \psi+r(1-\psi)-\kappa\left(\bar{\mu}-\mu_{t}\right)\left(\hat{E}+\hat{G} \mu_{t}\right) \\
& +\frac{\sigma_{\mu}^{2}}{2}\left\{-\left[\left(\hat{E}+\hat{G} \mu_{t}\right)^{2}+\hat{G}\right]+\left(\frac{1-\gamma-\phi}{1-\psi}+1\right)\left(\hat{E}+\hat{G} \mu_{t}\right)^{2}\right\}+\frac{1-\psi}{2(\gamma+\phi)}\left(\frac{\mu_{t}-r}{\sigma_{S}}\right)^{2} \\
& -\frac{1-\gamma-\phi}{\gamma+\phi}\left(\hat{E}+\hat{G} \mu_{t}\right) \rho \sigma_{\mu}\left(\frac{\mu_{t}-r}{\sigma_{S}}\right)+\frac{(1-\gamma-\phi)^{2}}{2(\gamma+\phi)(1-\psi)}\left(\hat{E}+\hat{G} \mu_{t}\right)^{2} \rho^{2} \sigma_{\mu}^{2} .
\end{aligned}
$$

Collecting terms in $\mu_{t}^{2}, \mu_{t}$ and constant terms, I can obtain $\hat{D}, \hat{E}$ and $\hat{G}$ as the solution to the following system of recursive equations:

$$
\begin{aligned}
0= & \frac{1-\psi}{2(\gamma+\phi) \sigma_{S}^{2}}+\left(\frac{k_{1}}{2}+\kappa-\frac{1-\gamma-\phi}{\gamma+\phi} \frac{\rho \sigma_{\mu}}{\sigma_{S}}\right) \hat{G} \\
& +\frac{\sigma_{\mu}^{2}}{2} \frac{1-\gamma-\phi}{1-\psi}\left(1+\frac{1-\gamma-\phi}{\gamma+\phi} \rho^{2}\right) \hat{G}^{2} \\
0= & -\frac{1-\psi}{\gamma+\phi} \frac{r}{\sigma_{S}^{2}}-\left(\kappa \bar{\mu}+\frac{1-\gamma-\phi}{\gamma+\phi} \frac{\rho r \sigma_{\mu}}{\sigma_{S}}\right) \hat{G}+\left(\kappa+k_{1}\right) \hat{E} \\
& +\left\{\frac{1-\gamma-\phi}{\gamma+\phi} \frac{\rho \sigma_{\mu}}{\sigma_{S}}+\sigma_{\mu}^{2}\left[1+\frac{1-\gamma-\phi}{1-\psi}\left(1+\frac{1-\gamma-\phi}{\gamma+\phi} \rho^{2}\right)\right] \hat{G}\right\} \hat{E} \\
0= & k_{1} \hat{D}-k_{0}-k_{1} \psi \log \beta+\beta \psi+r(1-\psi)+\frac{1-\psi}{2(\gamma+\phi)} \frac{r^{2}}{\sigma_{S}^{2}}-\frac{\sigma_{\mu}^{2}}{2} \hat{G} \\
& +\frac{\sigma_{\mu}^{2}}{2} \frac{1-\gamma-\phi}{1-\psi}\left(1+\frac{1-\gamma-\phi}{\gamma+\phi} \rho^{2}\right) \hat{E}^{2}+\left(\frac{1-\gamma-\phi}{\gamma+\phi} \frac{\rho r \sigma_{\mu}}{\sigma_{S}}-\kappa \bar{\mu}\right) \hat{E}
\end{aligned}
$$

where the solution to $\hat{G}$ is the root of the quadratic equation 28 that is associated with the positive root of the discriminant of the equation. The solutions to $\hat{D}$ and $\hat{E}$ follow immediately.

To obtain the optimal solutions to $\hat{D}, \hat{E}$ and $\hat{G}$, I use a numerical procedure suggested in Campbell and Viceira (1999). I first set the exponentiated mean $\log$ consumption-wealth ratio $\left(k_{1}\right)$ to $\beta$, which is the exact optimal solution for $\psi=1$ and then find the solutions of $\{\hat{D}, \hat{E}, \hat{G}\}$ given this value of $k_{1}$. These solutions imply a new value of $k_{1}$, for which a new set of solutions to $\hat{D}, \hat{E}$ and $\hat{G}$ is computed. This recursion proceeds until the absolute value of the difference between two consecutive values of $k_{1}$ is less than a small tolerance value. Finally, given the solutions to $\hat{D}, \hat{E}$ and $\hat{G}$, the optimal portfolio 
rule is

$$
\alpha_{t}=\frac{\mu_{t}-r}{(\gamma+\phi) \sigma_{S}^{2}}-\frac{1}{1-\psi} \frac{1-\gamma-\phi}{\gamma+\phi} \frac{\rho \sigma_{\mu}}{\sigma_{S}}\left(\hat{E}+\hat{G} \mu_{t}\right)
$$

The results shown above also nest the case of $\gamma=1$, for which the preference parameter $\eta$ when $\gamma=1, \eta_{\gamma \rightarrow 1}$, is given by

$$
\eta_{\gamma \rightarrow 1}=\lim _{\gamma \rightarrow 1} \frac{\phi}{W^{1-\gamma} \exp \left[-\frac{1-\gamma}{1-\psi}\left(\hat{D}+\hat{E} \mu_{t}+\frac{\hat{G}}{2} \mu_{t}^{2}\right)\right]}=\phi
$$

One can show that the HJB equation (9) can be solved by the value function of the form

$$
V\left(W_{t}, \mu_{t}\right)=\log W-\frac{1}{1-\psi}\left(\hat{D}+\hat{E} \mu_{t}+\frac{\hat{G}}{2} \mu_{t}^{2}\right)
$$

together with a loglinear approximation. The optimal values of $\hat{D}, \hat{E}$ and $\hat{G}$ can be solved by iterating the system of equations with $\gamma=1$. The optimal portfolio rule is given by

$$
\alpha_{t}=\frac{\mu_{t}-r}{(1+\phi) \sigma_{S}^{2}}+\frac{1}{1-\psi} \frac{\phi}{1+\phi} \frac{\rho \sigma_{\mu}}{\sigma_{S}}\left(\hat{E}+\hat{G} \mu_{t}\right) .
$$

\section{Proof of Proposition 1}

Proof. Assume that the value function takes the form

$$
V\left(W_{t}, \mu_{t}\right)=\left(A\left(\mu_{t}\right)\right)^{1-\gamma} \frac{W_{t}^{1-\gamma}}{1-\gamma} .
$$

The first term in Equation (9) becomes

$$
\begin{aligned}
& f(C, V)=\frac{\beta}{1-\frac{1}{\psi}} W^{1-\gamma}(A(\mu))^{1-\gamma}\left[\beta^{\psi-1}(A(\mu))^{1-1 / \psi}-1\right] \quad \text { when } \psi \neq 1 \\
& f(C, V)=\beta W^{1-\gamma}(A(\mu))^{1-\gamma}[\ln \beta-\ln (A(\mu))] \quad \text { when } \psi=1
\end{aligned}
$$


where I use the first order conditions $(10)$ and (11). Similarly, one can show that the second term in (9) is given by

$$
\begin{aligned}
\mathcal{D}^{(C, \alpha)} V= & W^{1-\gamma}(A(\mu))^{1-\gamma}\left\{\left[r+\alpha(\mu-r)-\beta^{\psi}(A(\mu))^{1-\psi}\right]+\frac{A^{\prime}(\mu)}{A(\mu)} \kappa(\bar{\mu}-\mu)\right. \\
& \left.-\frac{1}{2} \gamma \alpha^{2} \sigma_{S}^{2}+(1-\gamma) \frac{A^{\prime}(\mu)}{A(\mu)} \alpha \rho \sigma_{S} \sigma_{\mu}+\frac{1}{2}\left[-\gamma\left(\frac{A^{\prime}(\mu)}{A(\mu)}\right)^{2}+\frac{A^{\prime \prime}(\mu)}{A(\mu)}\right] \sigma_{\mu}^{2}\right\}
\end{aligned}
$$

and the last term in $(9)$ is given by

$$
W^{1-\gamma}(A(\mu))^{1-\gamma}\left\{-\frac{1}{2} \phi \alpha^{2} \sigma_{S}^{2}-\phi \frac{A^{\prime}(\mu)}{A(\mu)} \alpha \rho \sigma_{S} \sigma_{\mu}-\frac{1}{2} \phi\left(\frac{A^{\prime}(\mu)}{A(\mu)}\right)^{2} \sigma_{\mu}^{2}\right\} .
$$

Combining the above equations, one can rewrite $(9)$ in terms of the following ODE for $A(\mu)$

$$
\begin{aligned}
0= & \frac{\beta}{1-\frac{1}{\psi}}\left[\beta^{\psi-1}(A(\mu))^{1-1 / \psi}-1\right]+\left[r+\alpha^{*}(\mu-r)-\beta^{\psi}(A(\mu))^{1-\psi}\right] \\
& +\frac{A^{\prime}(\mu)}{A(\mu)} \kappa(\bar{\mu}-\mu)-\frac{1}{2}(\gamma+\phi)\left(\alpha^{*}\right)^{2} \sigma_{S}^{2}+[1-(\gamma+\phi)] \frac{A^{\prime}(\mu)}{A(\mu)} \alpha^{*} \rho \sigma_{S} \sigma_{\mu} \\
& +\frac{1}{2}\left[-(\gamma+\phi)\left(\frac{A^{\prime}(\mu)}{A(\mu)}\right)^{2}+\frac{A^{\prime \prime}(\mu)}{A(\mu)}\right] \sigma_{\mu}^{2}
\end{aligned}
$$

where the first term becomes $\beta[\ln \beta-\ln (A(\mu))]$ if $\psi=1$ and the optimal portfolio $\alpha^{*}$ is given by ${ }^{12}$

$$
\alpha_{t}^{*}=\frac{\mu_{t}-r}{(\gamma+\phi) \sigma_{S}^{2}}+\frac{1-\gamma-\phi}{\gamma+\phi} \frac{A^{\prime}(\mu)}{A(\mu)} \frac{\rho \sigma_{\mu}}{\sigma_{S}} .
$$

Note that the solution to $A(\mu)$ depends on $\phi$ only through $\gamma+\phi$. Thus, observational equivalence holds. This completes the proof.

\footnotetext{
${ }^{12} \overline{\text { I use the first order condition } \sqrt{12} \text { for portfolio }}$ choice.
} 


\section{References}

[1] Ai, H., 2007. "Information about long-run risks: asset pricing implications." Journal of Finance forthcoming.

[2] Anderson, E. W., L. P. Hansen, and T. J. Sargent (2000). "Robustness, detection, and the price of risk. "Working paper University of Chicago.

[3] Anderson, E. W., L. P. Hansen, and T. J. Sargent (2003). "A semigroups for model specification, robustness, price of risk, and model detection." Journal of the European Economic Association $1,68-123$.

[4] Bansal, R. and A. Yaron (2004). "Risks for the long run: a potential resolution of asset pricing puzzles." Journal of Finance 59, 1481-1509.

[5] Bhamra, H. and R. Uppal (2006). "The role of risk aversion and intertemporal substitution in dynamic consumption-portfolio choice with recursive utility. "Journal of Economic Dynamics and Control 30, 967-991.

[6] Campbell, J. Y. and L. M. Viceira (1999). "Consumption and portfolio decisions when expected returns are time varying." Quarterly Journal of Economics 114, 433-495.

[7] Campbell, J.Y., G. Chacko, J. Rodriguez and L. M. Viceira (2004)"Strategic asset allocation in a continuous-time var model." Journal of Economic Dynamics and Control 28 2195-2214.

[8] Chacko, G. and L. M. Viceira (2005). "Dynamic consumption and portfolio choice with stochastic volatility in incomplete markets." Review of Financial Studies 18, 1369-1402.

[9] Chen, Z. and L. G. Epstein (2002). "Ambiguity, risk and asset returns in continuous time." Econometrica 4, 1403-1445.

[10] Duffie, D. and Epstein, L.G. (1992). "Stochastic differential utility." Econometrica 60, 353-394. 
[11] Epstein, L. G. and M. Schneider (2007). "Learning under ambiguity." Review of Economic Studies $74,1275-1303$.

[12] Gilboa, I. and D. Schmeidler (1989). "Maxmin expected utility with non-unique priors." Journal of Mathematical Economics 18, 141-153.

[13] Hansen, L. P., and T. J. Sargent (2001). "Robust control and model uncertainty." American Economic Review 91, 60-66.

[14] Hansen, L. P., T. J. Sargent, G. A. Turmuhambetova, and N. Williams (2006). "Robust control, min-max expected utility, and model misspecification". Journal of Economic Theory 128, 45-90.

[15] Kim, T. S., and E. Omberg (1996). "Dynamic nonmyopic portfolio behavior." Review of Financial Studies 9, 141-161.

[16] Kreps, D. M. and E. L. Porteus, (1978). "Temporal resolution of uncertainty and dynamic choice." Econometrica 46, 185-200.

[17] Maenhout, P. (2004). "Robust portfolio rules and asset pricing. Review of Financial Studies."17, 951-983.

[18] Maenhout, P. (2006). "Robust portfolio rules and detection-error probabilities for a meanreverting risk premium." Journal of Economic Theory 128, 136-163.

[19] Merton, R. C. (1971). "Optimal consumption and portfolio rules in a continuous-time model." Journal of Economic Theory 3, 373-413.

[20] Samuelson, P. (1969). "Lifetime portfolio selection by dynamic stochastic programming." Review of Economics and Statistics 51, 239-246.

[21] Schroder M., and C. Skiadas (1999). "Optimal consumption and portfolio selection with stochastic differential utility." Journal of Economic Theory 89, 68-126. 
[22] Schroder M., and C. Skiadas (2003). "Optimal lifetime consumption-portfolio strategies under trading constraints and generalized recursive preferences." Stochastic Processes and their Applications 108, 155-202.

[23] Uppal, R., and T. Wang (2003). "Model misspecification and underdiversification." Journal of Finance 58, 2465-2486.

[24] Vissing-Jørgensen and Attanasio (2003). "Stock-market participation, intertemporal substitution, and risk-aversion." American Economic Review 93, 383-391.

[25] Wachter, J. (2002). "Portfolio and consumption decisions under mean-reverting returns: an exact solution for complete markets." Journal of Financial and Quantitative Analysis 37, 63-91. 
Table 1: This table presents the parameter estimates used in the calibration analysis. The estimates are drawn from Campbell et al. (2004) for the quarterly data from 1947:1-1995:4. Campbell et al. (2004) describes the details of recovering continuous-time estimates from discrete-time estimates.

\begin{tabular}{lr}
\hline \hline Parameter description & Parameter values \\
\hline Quarterly sample $(1947: 1-1995: 4)$ & \\
Rate of time preference $\beta$ & 0.0153 \\
Riskless interest rate $r$ & 0.0082 \\
Volatility of stock return $\sigma$ & 0.0790 \\
Volatility of expected return $\sigma_{\mu}$ & 0.0057 \\
Mean reversion parameter $\kappa$ & 0.0439 \\
Unconditional mean of expected return $\bar{\mu}$ & 0.0213 \\
Correlation between stock return and expected return $\rho$ & -0.9626 \\
\hline \hline
\end{tabular}




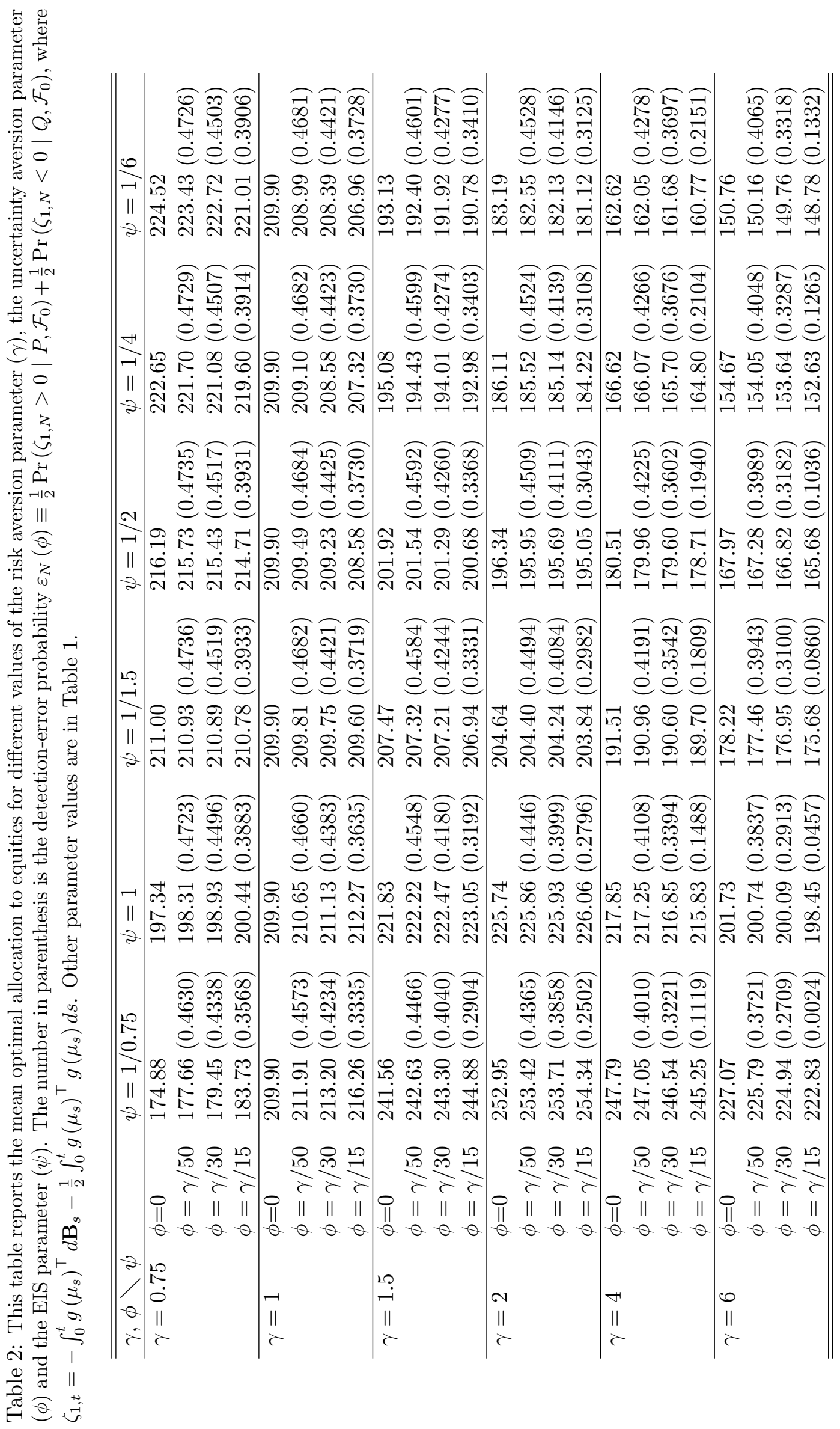



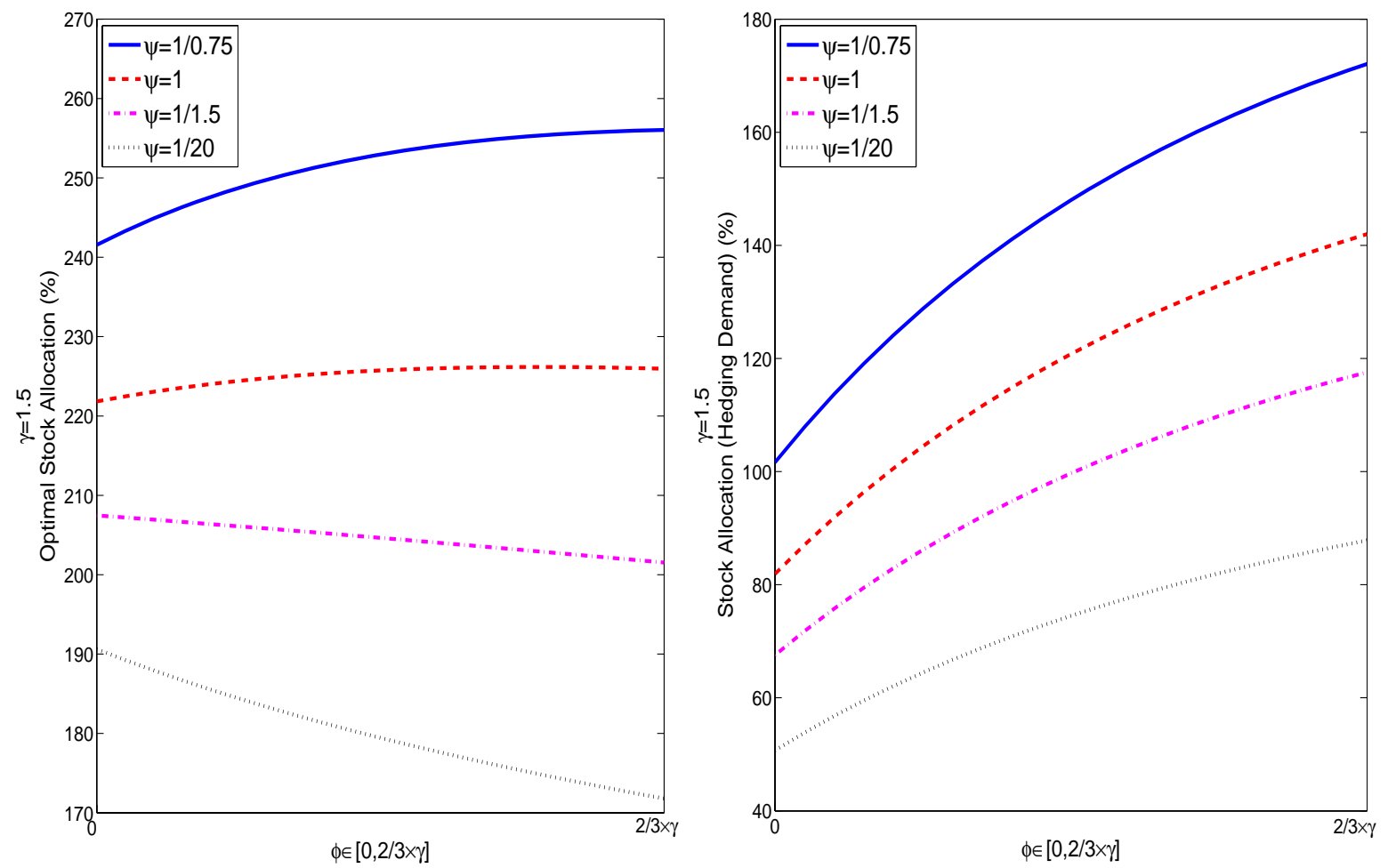

Figure 1: This figure plots the optimal proportion of wealth allocated to equities (left panel) and the proportion allocated to equities due to hedging demand (right panel) for different values of $\phi \in\left[0, \frac{2}{3} \gamma\right]$. The risk aversion parameter $\gamma$ is equal to 1.5 . The EIS parameter $\psi$ takes four values, $1 / 0.75,1,1 / 1.5$ and $1 / 20$. Other parameter values are in Table 1. 